\title{
Le projet de loi antiterroriste canadien : une sélection sécuritaire
}

\author{
Pascal Dominique-Legault \\ Université Laval, Département de sociologie
}

La manière dont est défini le terrorisme en tant que problème public détermine la manière de concevoir les solutions à ce problème. Ainsi, la perception que des mesures sécuritaires sont adéquates, nécessaires, efficaces, proportionnelles ou, au contraire, qu'elles sont abusives ou déséquilibrées, est largement tributaire d'un processus définitionnel. Dans cet article, nous identifions comment les parlementaires canadiens ont défini le problème du terrorisme dans le cadre des débats sur le projet de loi antiterroriste canadien, qui ont eu lieu du 15 octobre 2001 au 18 décembre 2001. Plus encore, nous examinons l'impact que ces différentes constructions discursives a eu sur ce que les solutions préconisées par les parlementaires, solutions qui transparaissent dans les mesures proposées par et dans le projet de loi. Cet article traite ainsi de la sélection sécuritaire qui s'est opérée dans le projet de loi $C-36$, c'est-à-dire de la stratégie sécuritaire générale qui a été priorisée. Nous qualifierons la forme particulière que prend cette sélection en la situant au sein des alternatives proposées qui ont été écartées, avec pour objectif de mieux comprendre ce choix sécuritaire et ses implications. Au final, seront proposées des pistes de réflexion sur les effets de pouvoir des discours sélectionnés et institués dans le projet de loi antiterroriste canadien.

Mots clés : discours, parlementaires, terrorisme, construction sociale, Canada

The way in which terrorism is defined as a public problem determines how solutions are designed to address this problem. Thus, the perception that security measures are adequate, necessary, effective, proportional or how they are perceived unfair or unbalanced, is largely dependent on a definitional process. In this paper, we identify how Canadian parliamentarians have defined the problem of terrorism in the context of the discussions on the proposed Canadian Anti-Terrorism Bill, which took place from October $15^{\text {th }}, 2001$ to December $18^{\text {th }}$, 2001. Furthermore, we examine the impact that these different discursive constructions had on the solutions proposed by parliamentarians, and on the solutions that are reflected in the proposed measures and the Bill. Therefore, this article discusses the selection process that took place in the creation of Bill C-36, and thus discusses the overall security strategy that has been prioritized in it. We describe the particular form of this selection by placing it within the proposed alternatives that were rejected, with the aim of better understanding the choices made in the creation of Bill $C$ 36 and their implications. We will conclude with a discussion on the effects of power of the discourses that were chosen and instituted in the Canadian Anti-Terrorism Bill.

Keywords: discourse, parliamentary, terrorism, social construction, Canada

Ce n'est pas d'aujourd'hui que la criminologie à perspective critique s'intéresse aux processus de criminalisation primaire, soit aux différentes forces qui s'affrontent dans la production d'une norme pénale (Landreville, 1986). Dans une lignée théorique similaire à celle avancée par la criminologie critique s'établissent, depuis les années 1990, les « études critiques de la sécurité » qui privilégient, à différents degrés, des approches critiques et/ou constructivistes à la sécurité (Macleod, 2004; Moffette, 2012). Ces études comprennent «l'École de Copenhague » qui se rassemble principalement autour des contributions théoriques de Barry Buzan et d'Ole Weaver sur « les processus de sécurisation ». Cette « école » s'intéresse 
à l'étude des constructions discursives des enjeux de sécurité et des processus par lesquels des élites politiques cadrent des situations en enjeux sécuritaires. Ces élites chercheraient, par le discours et à travers un processus complexe, à légitimer l'octroi de pouvoirs considérables à l'État par des tentatives de « sécurisation », dans un contexte donné. La réussite des processus de sécurisation dépend notamment de l'acceptation des discours sécuritaires par l'auditoire. "L'École de Paris », inspirée de concepts foucaldiens, s'inscrit aussi dans ces études critiques de la sécurité, mais complexifie l'analyse de «l'École de Copenhague » en priorisant les effets des discours et les relations de pouvoir entre les acteurs. En plus de s'intéresser aux dispositifs de « sécurisation » et aux discours, cette école, notamment incarnée par Didier Bigo, Jef Huysmans et Bill McSweeney, cherche à replacer l'analyse « (...) vers [la] multitude de pratiques situées qui forment et appliquent une variété de techniques de gouvernement modulant la conduite de la liberté en situations d'insécurité. » (Huysmans, 2006 : 43, trad. par Mofette, 2012) Cette « école » cherche à replacer les discours de sécurisation à l'intérieur de l'univers complexe des relations de pouvoir entre acteurs et des «luttes » de discours, plutôt que de considérer ces discours selon la forme d'émetteurrécepteur linéaire typique de la pensée de Weaver (Macleod, 2004; Moffette, 2012).

C'est dans une telle perspective d'étude critique de la sécurité que cet article se propose d'analyser les discours de parlementaires canadiens prononcés lors de l'adoption du projet de loi antiterroriste canadien en 2001. Plus spécifiquement, il se propose de comprendre comment ce projet de loi incarne une sélection sécuritaire particulière. Sachant que la définition d'un problème limite et encadre les réponses formulées (Delumeau, 1978; Beauchamp, 1996), la manière d'articuler la menace terroriste circonscrit, elle aussi, les solutions et réponses privilégiées pour y répondre. Ainsi, la perception que des mesures sécuritaires sont adéquates, nécessaires, efficaces ou proportionnelles pour répondre à la menace terroriste, ou à l'inverse, qu'elles sont abusives ou déséquilibrées, est largement tributaire de la construction discursive du problème. Â cet égard, les débats parlementaires lors de l'adoption de projets de loi antiterroristes constituent un lieu fort intéressant pour observer ces différentes constructions discursives de problèmes sécuritaires. Le droit, en étant un champ de bataille politique de l'incertitude, en arrive, par un processus complexe, à institutionnaliser une seule version de l'analyse du problème, en l'occurrence ici le terrorisme, malgré les divergences entre partis politiques et témoins experts prenant part au processus législatif. « This power of assigning privilege in each case decision inevitably serves some interests and not others. For the unprivileged, it is a power that hurts, causes resentment, provokes a sense of injustice, produces insecurity, and poses again the problem of uncertainty » (Ericson, 2007: 205-6). Dans cet article, le projet de loi antiterroriste canadien (C-36) servira d'exemple concret d'une solution sécuritaire proposée par le gouvernement libéral majoritaire de l'époque en réponse aux attentats terroristes du 11 septembre 2001.

Cette recherche contribue à la littérature scientifique portant sur le terrorisme : (1) en offrant une analyse d'un contenu canadien en matière de terrorisme et de lutte antiterroriste; (2) en appliquant une approche théorique critique de la sécurité qui privilégie une analyse discursive des constructions sociales du problème du terrorisme formulées par les parlementaires canadiens, des rapports de force qui permettent l'institutionnalisation d'une stratégie sécuritaire spécifique permettant ultimement de penser le problème et les solutions écartées; et (3) en fournissant une première analyse des discours parlementaires sur le terrorisme pendant l'adoption du projet de loi antiterroriste canadien. En effet, bien qu'un recueil important d'articles portant sur les mesures sécuritaires prévues dans la loi antiterroriste ait été rédigé en 2001 (The Security of Freedom : Essays on Canada's Anti-Terrorism Bill), il ne fournit aucune véritable analyse des discours politiques prononcés durant le processus législatif, présentant principalement des articles rédigés pour une conférence qui a eu lieu avant que ne soit adopté C-36 à l'étape du comité parlementaire de la Chambre des communes. Notre article constitue donc, à notre connaissance, la première recherche portant sur les discours des parlementaires canadiens prononcés lors de l'adoption du projet de loi antiterroriste canadien.

Ainsi, à l'aide d'une analyse documentaire des discours politiques officiels transcrits par la Chambre des communes et le Sénat du Canada, nous identifierons comment les parlementaires canadiens ont défini la menace terroriste dans le cadre des débats sur le projet de loi antiterroriste canadien qui ont eu lieu du 15 octobre 2001 au 18 décembre 2001. Nous préciserons par la suite les impacts que ces définitions de la menace ont eus sur ce que certains parlementaires ont présentés comme des solutions équilibrées ou 
justifiées au terrorisme. Nous exposerons ainsi la sélection « sécuritaire » qui en découle, soit le choix stratégique sécuritaire général qui a été priorisé pour " assurer » la sécurité nationale avec C-36 et, par conséquent, les alternatives qui ont été laissées dans l'ombre lors de cette sélection. En dernier lieu, nous proposons des pistes de réflexion sur les effets de pouvoir que peuvent entretenir ces discours sélectionnés. Cela nous permettra de situer les pratiques sécuritaires canadiennes actuelles. Toutefois, avant de faire l'état de ces sélections sécuritaires, nous commençons par exposer le contexte dans lequel la menace terroriste a été érigée en priorité de l'agenda politique de plusieurs pays comme le Canada.

\section{Le politique et la sélection des risques}

Les attentats du 11 septembre 2001 ont poussé spectaculairement la lutte contre le terrorisme en tête des agendas politiques de nombreux pays occidentaux, permettant la mise en place d'une panoplie de mesures sécuritaires. Selon Ericson (2007), l'obsession contemporaine à diminuer les risques au sein des démocraties néolibérales aurait favorisé ce processus. Les sécurités économique, sociale, nationale et domestique, qui figurent au sommet des agendas politiques, encouragent des investissements massifs au niveau de l'évaluation du risque et, trouvant aisément leurs échos dans les médias, alimentent paradoxalement l'insécurité et les perceptions du danger dans la population, et donc les demandes pour augmenter la sécurité dans différents domaines (2007 : 1). Cette insécurité perpétuée par la constante réponse sécuritaire est confirmée par Douglas et Wildavsky (1983) qui soutiennent que la simple perception du risque est suffisante pour engendrer le risque. Alors, par la surintellectualisation du processus de décision et par l'exagération d'obstacles irrationnels, particulièrement par le relais médiatique, on arrive à produire nous-même de l'insécurité. Une telle analyse fait résonance avec les positions des tenants de «l'École de Copenhague » qui soutiennent que « (...) les discours ont une capacité performative importante capable d'objectiver la réalité » (Macleod, 2004; Moffette, 2012 : 44).

Si dans nos démocraties occidentales nous avons tendance à tolérer de moins en moins l'incertitude dans toutes les sphères de la vie, Ericson nous dit que c'est particulièrement le cas face la menace terroriste nouvellement priorisée. Le savoir scientifique serait utilisé moins comme source de certitude et plus fréquemment comme source d'incertitude pour prévenir les risques et essayer de contrôler ou d'atténuer ces derniers (2007 : 204). Similairement, Beauchamp est d'avis que beaucoup ont davantage tendance à éviter le risque plutôt que de l'assumer (1996 : 20). Mais avant de poursuivre, clarifions ce que Beauchamp entend par les concepts de danger (ou de menace), de peur et de risque. Le danger c'est la réalité extérieure liée à la précarité de l'existence humaine et les menaces objectives qui pèsent sur elle. La peur, quant à elle, c'est ce qui est ressenti par l'humain qui se trouve face au danger qu'il perçoit. Et lorsqu'on parle de risque, c'est la mesure du danger qui est calculée, étudiée et prévue (1996:171). Le risque émane souvent d'un contexte d'incertitude et d'angoisse qui l'amène à jouer le rôle d'un carrefour qui oblige une décision : soit celle de risquer quelque chose au nom de valeurs ou de ne pas risquer au nom d'autres valeurs (1996: 20).

Il importe également de comprendre que le risque n'est pas quelque chose d'objectif en soi : tout un processus de sélection régit les risques au niveau individuel, mais également au niveau collectif et donc au niveau de l'État (1996:23). Le risque fait partie de la rhétorique politique et, comme nous l'avons vu, certaines élites politiques cherchent, selon « l'École de Copenhague ", à nous « sécuriser » en cadrant des situations pour en faire des enjeux sécuritaires qui légitimeraient des pouvoirs supplémentaires. Dans cet ordre d'idées, Mueller (2006) et Kassimeris (2007) ont tous deux démontré comment la présentation de la problématique du terrorisme pouvait être utilisée pour faire des gains politiques. Avec la quantité de dangers qui pèse sur l'être humain, il est clair que ce dernier ne peut prendre tous les risques au sérieux sans devenir terrorisé et non fonctionnel (Beauchamp, 1996: 22). Alors, d'un individu et d'une société à l'autre, une sélection des risques s'effectue, consciemment ou inconsciemment, par un processus complexe. Dans cette perspective, nous soutenons, suivant en cela les affirmations de Beauchamp, que «[...] chaque culture sélectionne et privilégie des risques, se fait une représentation de la bravoure et de la couardise, du risque à affronter et du risque à fuir. » (1996:23).

Ainsi, le risque qui est mobilisé dans un discours parlementaire n'a rien d'absolu. Le discours sert à sélectionner certains risques plutôt que d'autres, à blâmer certaines entités plutôt que d'autres. Certains 
risques sont mis en évidence lorsque d'autres sont occultés. C'est ainsi que nous définissons le projet de loi antiterroriste canadien comme une institutionnalisation d'une sélection qui est le résultat d'un processus où différents acteurs s'affrontent de manière discursive et parviennent à mobiliser différemment le risque. Chacun s'efforce de convaincre un auditoire de croire (ou pas) en cet enjeu sécuritaire construit et de faire accepter (ou pas) des pouvoirs supplémentaires pour gérer le risque. Il faut dire que de nos jours, nous avons tendance à utiliser à profusion les institutions sociales, les forces de l'ordre, les lois, les droits, l'armée et les assurances pour se rassurer et se protéger des peurs collectives. "Quand le niveau de peur augmente, nous exigeons de l'État des protections accrues : une répression plus grande à l'égard des contrevenants (emprisonnements plus longs, peine de mort), un meilleur contrôle des frontières, moins d'immigration. [...] Devant les grands cataclysmes, la rumeur invente des causes, parfois des boucs émissaires, et demande des actions spectaculaires. » (Beauchamp, $1996: 28$ ).

Ainsi, autant l'adoption d'une loi permet d'exprimer le mythe de la certitude à court terme, en nommant un risque, en l'expliquant, en diabolisant certains acteurs et en prévoyant des solutions, autant la loi peut contribuer à l'incertitude, en l'incubant et en manufacturant des incertitudes (Ericson, 2007 : 206). Même si ces lois que nous adoptons offrent la promesse de la certitude et de la sécurité à court terme, il faut comprendre, à l'instar d'Ericson, qu'elles ont un mandat impossible à long terme, car des dangers catastrophiques sont inévitables et chaque évènement "terroriste » confirme les limites d'un tel régime, qu'Ericson nomme de « gouvernance par le crime » (2007 : 219). L'un des effets pervers d'un tel régime, qui veut gouverner l'incertitude le plus efficacement possible, réside non seulement dans la désignation de « responsables » de l'acte terroriste, mais dans la désignation de « responsables », au sein des structures de gestion du risque crées, qui ont manqué à leur devoir de prévoir et de gouverner le futur adéquatement. On peut aisément comprendre la position délicate des gestionnaires de l'État et des agences sécuritaires, qui, à la suite d'un attentat, pourraient être rapidement mis sur la sellette pour ne pas avoir, aux yeux de certains, efficacement géré le risque.

Au Canada comme ailleurs, nous avons vu le terrorisme se hisser à la tête des agendas politiques. Avant les attentats du 11 septembre 2001, le Canada était déjà dans une période de transformation institutionnelle sécuritaire initiée et entretenue notamment par la chute du communisme, la globalisation des marchés, l'intensification de la concurrence internationale, l'explosion et la miniaturisation des technologies de communication qui ont généré une forte concurrence entre les services de renseignement et de sécurité (SRS), les organes de presse et les services de renseignements privés (Baud, 2000). En effet, la perception politique de ce qui constituait une menace à la sécurité nationale était en train de se redéfinir lentement et graduellement, comme dans les autres pays, lorsque les attentats terroristes américains en 2001 sont venus accélérer le processus dans son ensemble. La menace terroriste a donc remplacé la menace communiste comme cible dominante des institutions chargées d'assurer la sécurité nationale et ce, à l'intérieur d'un nouvel environnement mondialisé avec toutes ses complexités (Baud, 2000; Brodeur, 2003). À ce sujet, Bigo suggère même que se constitue un discours dominant qui présente des insécurités mondiales et qui cherche à nous sécuriser contre elles par une mondialisation (ou une transnationalisation) de la sécurité (2005).

C'est dans cette logique que des initiatives sécuritaires diverses sont développées au Canada et que des ressources sont attribuées à différents organismes sécuritaires (pour le détail de ces initiatives après les attentats du 11 septembre 2001, voir : Wark, 2004). Le projet de loi antiterroriste canadien (C-36) est, quant à lui, soumis à la Chambre des Communes en première lecture le 15 octobre 2001, soit seulement 34 jours après les attentats meurtriers de New-York par le gouvernement libéral, alors majoritaire. C-36 obtient l'approbation de la législature et la sanction royale le 18 décembre 2001, soit 61 jours après sa soumission à la Chambre des communes. À première vue, ceci peut sembler un délai plus raisonnable que les lois antiterroristes française (16 jours), britannique (32 jours) et allemande (36 jours) (Haubrich, 2003 : 19). Cependant, il s'agit tout de même d'un court délai pour une pièce législative de 175 pages qui propose des amendements à plus de dix lois différentes et qui vise la ratification de deux traités onusiens : soit la Convention internationale pour la répression des attentats terroristes à l'explosif de 1997 et la Convention internationale pour la répression du financement du terrorisme de 1999. Il faut comprendre que le contexte des débats politiques sur C-36 en est un (1) de pressions sécuritaires domestiques (lié au sentiment 
d'insécurité au pays); (2) de pressions sécuritaires internationales (lié à la ratification des dites conventions internationales, au sentiment d'insécurité de nos alliés sur la scène international, mais également lié à l'importance de choisir son camp dans le cadre de la «guerre contre le terrorisme» qui venait d'être déclenchée par le président George W. Bush avec sa formulation : «(e)ither you are with us, or you are with the terrorists » (White House, 20-09-2001)); mais également (3) de pressions internationales économiques occasionnées par le ralentissement des échanges à la frontière entre le Canada et les États-Unis suite aux attentats.

Tel que souligné par Gross (2001), les perceptions d'une menace physique substantielle, le sentiment d'insécurité croissant d'être une victime potentielle, les sentiments de frustration et de haine envers les terroristes responsables des attentats du 11 septembre combinés à une interprétation que les restrictions seront plus ou moins temporaires et que la majorité des mesures viseront des gens de l'extérieur ( outsiders »), ont certainement pu encourager une adoption législative rapide qui laisse place davantage aux émotions qu'aux analyses calculées et rationnelles. À ceci s'est ajouté l'argumentaire gouvernemental qui insistait sur le fait que la Loi antiterroriste était conforme à la Charte canadienne des droits et libertés; qu'elle était devenue nécessaire pour remplir nos obligations internationales dans le cadre de plusieurs conventions (nommées ci-haut); que plusieurs alliés du Canada avaient adopté des dispositions similaires dans leurs propres législations domestiques; et enfin que cette nouvelle loi offrait plus de garanties et de protections aux droits et libertés que l'ancienne Loi sur les mesures de guerre (Weinrib, 2001).

Malgré ces argumentaires de « sécurisation » libéraux, l'analyse des débats parlementaires met en évidence la difficulté à parvenir à un consensus sur un sujet central, celui de l'équilibre entre un idéal de sécurité et les droits et libertés. Il est clair que tous les parlementaires prônent un équilibre entre ces deux idéaux; cependant, leurs conceptions des mesures sécuritaires qui sont adéquates, nécessaires, efficaces, proportionnelles, voire abusives, diffèrent. Nous soutenons que ces divergences naissent, en grande partie, des divergences dans la construction discursive du problème du terrorisme.

\section{Une sélection « sécuritaire »}

Maintenant que nous comprenons en quoi le projet de loi antiterroriste canadien est une sélection sécuritaire d'un risque particulier parmi tant d'autres en société, nous allons qualifier la sélection qu'elle constitue en analysant les discours des parlementaires canadiens sur le terrorisme. Notons qu'au moment des débats, cinq partis politiques s'affrontaient : le Parti libéral, l'Alliance canadienne, la Coalition du Parti progressiste-conservateur / Caucus de la représentation démocratique (PC/RD), le Bloc québécois (BQ) et le Nouveau parti démocratique (NPD). Notre analyse est basée sur une approche inductive combinée à la grille d'analyse élaborée par Martin, Fichelet et Fichelet (1977) qui proposent un examen en trois temps : 1) l'identification de l'acteur du discours (qui?); 2) l'identification de son contenu (dit quoi?); 3) l'identification de la perspective qu'il privilégie soit (pour dire quoi?/dans quel but?). Notre analyse des discours parlementaires permet de confirmer notre prémisse de base qui sous-tend que la manière d'identifier les problèmes et les menaces à notre sécurité dicte, encadre et limite dans une large mesure les solutions prônées pour y faire face. En effet, en analysant le contenu et la teneur des débats, nous constatons que tous les parlementaires ne se représentent pas les « causes profondes » du terrorisme pareillement. Conséquemment, ils ne proposent pas les mêmes solutions et moyens pour y faire face.

En effet, tous les partis se représentent, à différents degrés, une menace terroriste essentiellement incarnée par des individus ou des groupes, parfois prêts à mourir pour leur cause, qui viennent davantage d'ailleurs que d'ici et qui se réfugient dans des pays libres comme le Canada, en utilisant de fausses identités et de faux papiers pour entrer au pays, afin d'orchestrer des attentats terroristes. C'est notamment sur une perception du problème permanent, non exceptionnel et global, incarné par des individus fanatiques entraînés dans des camps à l'étranger, que les Libéraux justifient le projet de loi C-36 qu'ils déposent à la Chambre des communes.

Nous protégeons nos valeurs et définissons la menace posée par le terrorisme aux nations libres et civilisées du monde. [...] Le projet de loi C-36 est un des éléments du plan d'action global 
du gouvernement du Canada en vue de préserver la sécurité de notre pays, un plan dont les objectifs sont d'empêcher les terroristes d'entrer au Canada et de protéger les citoyens contre les actes terroristes, de mettre en place les outils nécessaires pour identifier, poursuivre, condamner et punir les terroristes, de maintenir la sécurité aux frontières, de travailler avec la communauté internationale afin que les terroristes soient traduits en justice et de s'attaquer avec elle aux racines de la haine. [...] Que nous habitions en Amérique du Nord ou ailleurs dans le monde, le terrorisme représente une menace globale [...] Nous devons sans hésiter travailler avec nos voisins et nos alliés afin que ceux qui choisissent de terroriser des innocents et d'appuyer des terroristes comprennent que nous allons interrompre leurs sources de financement. Nous allons les trouver et nous allons les punir pour les actes de violence qu'ils ont posés (Anne McLellan, Libéral, Hansard 16-10-2001, p.6164-5).

C-36 est un projet de loi omnibus qui modifie une dizaine de lois, en crée une nouvelle et confère de nouveaux pouvoirs policiers et ministériels. On y retrouve notamment : la criminalisation non seulement de l'activité terroriste nouvellement définie, mais également de la facilitation d'une telle activité. On y ajoute le pouvoir policier de l'arrestation et de la détention préventive d'une personne soupçonnée d'avoir l'intention de commettre une telle activité, et ce, combiné avec un critère juridique (« le soupçon ») moins exigeant au niveau de la preuve que le traditionnel « motif raisonnable de croire ». Toute personne soupçonnée de détenir des informations sur de potentielles activités terroristes peut également se voir contrainte, par un juge, de comparaître et de répondre à des questions visant à recueillir des éléments de preuve. Cette loi définit également un pouvoir ministériel de délivrer un certificat interdisant la divulgation de toute information qui pourrait nuire à la sécurité nationale ou aux relations internationales, ainsi que celui de fournir, le cas échéant et pendant des poursuites judiciaires, un résumé des preuves plutôt que l'entièreté des preuves détenues. Le projet de loi C-36 prévoit aussi la «modernisation » de la Loi sur les secrets officiels. On y octroie, au gouverneur en conseil, le pouvoir d'établir une liste officielle d'entités terroristes et, au Centre de la sécurité des télécommunications Canada (CSTC), un accroissement des pouvoirs de surveillance électronique, notamment pour surveiller des transactions financières, pour assurer leur archivage et d'autres pouvoirs pour saisir, bloquer et confisquer les biens appartenant aux « terroristes ». On y prévoit le pouvoir ministériel de retirer le statut officiel d'organisme de bienfaisance dans certains cas et un certain pouvoir de prélever l'ADN des personnes reconnues coupables d'activité terroriste (à l'exception de ceux qui financent ces activités), etc. Ainsi, le projet de loi C-36, qui présente le discours libéral, semble être une solution qui transpose sur des individus la menace et la responsabilité, ce qui permet de justifier une solution qui prône, notamment, la criminalisation et une surveillance accrue d'individus directement impliqués dans une activité terroriste, ou de personnes soupçonnées d'aider et de financer des groupes terroristes.

L'analyse montre que plusieurs parlementaires ont davantage tendance à limiter leur définition des problèmes terroristes à ces individus et à leur réseau. C'est notamment le cas de l'Alliance canadienne et de la PC/RD, qui, rappelons-le, s'uniront en 2003 pour former le Parti conservateur du Canada. La PC/RD présente surtout une image terroriste qui met en scène des personnes extrémistes qui viennent d'ailleurs, qui veulent détruire les sociétés occidentales, qui s'entraînent pour leur mission et qui bénéficient d'un certain financement pour y arriver.

J'entends toujours dire que des personnes foncièrement mauvaises profitent de ces droits. Je songe aux gens qui, dès qu'ils mettent le pied au Canada, sont malheureusement couverts et protégés par notre charte des droits et libertés, qui a été mise en place avec les meilleures intentions du monde. Il y a des gens qui en abusent. Pour contrebalancer cela en ces temps de crise pour notre pays, il faudra empiéter sur les droits de ces individus (Jay Hill, PC/RD, Hansard 16-10-2001: 6219).

D'autres estiment, à l'image de Peter Goldring que : 
[L]es ennemis d'aujourd'hui sont des lâches qui se cachent dans des cavernes, derrière des rochers et qui bénéficient des libertés pour lesquelles nous nous sommes battus dans le passé. [...] Avec l'aide de nos alliés, nous viendrons à bout de ces terroristes. Nous démasquerons et nous traduirons en justice toutes les personnes déterminées à anéantir les efforts et les réussites de nos nations civilisées. Nous appliquerons une justice vengeresse à l'égard des coupables de ce lâche assassinat de milliers de civils pacifiques qui vaquaient simplement à leurs activités professionnelles pour faire vivre leur famille (Peter Goldring, AC, Hansard 16-10-2001: 6240-1).

Par conséquent, il n'est pas étonnant de voir l'Alliance canadienne prôner des " mesures législatives sévères et efficaces pour améliorer la sécurité nationale » (Vic Toews, AC, Hansard, 16-10-2001 : 6169) contre des individus qu'elle juge sans scrupules. Cela explique également pourquoi l'Alliance a offert son appui au gouvernement libéral présentant comme « raisonnables » deux des mesures libérales les plus controversées, soit les arrestations préventives et l'audience d'investigation (décrites précédemment). De plus, l'Alliance ajoute que «[...] l'obligation de respecter une procédure équitable même pendant les audiences d'investigation pourrait nuire à la divulgation de renseignements en temps opportun et nous empêcher de réagir rapidement à toute menace d'activité terroriste imminente » (Vic Toews, AC, Idem). Ainsi, en plus de montrer l'insuffisance du nouveau pouvoir conféré, l'Alliance canadienne a dénoncé le fait « [...] que le projet de loi ne refuse pas la libération conditionnelle aux terroristes reconnus coupables de meurtres multiples » (Vic Toews, Ibid. : 6168). Ce parti, clairement insatisfait de la sévérité des mesures et de l'étendue des pouvoirs prévues par le projet de loi C-36, a également accusé le gouvernement libéral d'avoir réagi trop lentement à la menace, d'avoir rejeté nombre de ses recommandations au cours des années passées et, depuis le 11 septembre 2001, de ne pas avoir criminalisé, dans sa série de nouvelles infractions, " l'appartenance » à un groupe terroriste comme l'a fait le Canada dans le projet de loi C-24 sur le crime organisé. De plus, l'Alliance canadienne a critiqué le gouvernement libéral pour ne pas avoir inclus de dispositions prévoyant un octroi annuel automatique et obligatoire de ressources destinées aux services de police et de sécurité de première ligne afin d' « [...] être en mesure de réagir face aux ressources, aux fonds et aux connaissances technologiques quasi illimités des organisations terroristes » (Vic Toews, AC, Ibid. : 6169). Elle s'est en outre plainte que le projet de loi C-36 ne contenait aucune mesure pour expulser les réfugiés, les personnes soupçonnées de terrorisme, ou encore, les gens qui menacent la sécurité du Canada. Stockwell Day a enfin réclamé « [...] le pouvoir de détenir les demandeurs d'asile qui arrivent au Canada sans papiers d'identité ou que nous avons des motifs raisonnables de croire associés au terrorisme ou au crime organisé » (Stockwell Day, AC, Ibid.: 6209). Somme toute, selon l'Alliance canadienne, « [1]e gouvernement a finalement répondu de façon significative à la menace que constitue le terrorisme international, mais il reste beaucoup à faire. Les députés de l'Alliance canadienne, qui forment l'opposition, sont déterminés à s'assurer avec le gouvernement que les mesures nécessaires soient prises pour protéger la paix et la sécurité au Canada » (Vic Toews, AC, Ibid. : 6170). Comme solution, ces parlementaires, qui définissent le terrorisme comme un problème essentiellement individuel et centré sur quelques extrémistes de l'extérieur, ont davantage tendance à accepter le sacrifice ou la réduction des droits et libertés des Canadiens au nom de la sécurité nationale.

Il n'est pas étonnant de constater que la PC/RD adopte des définitions de la menace et des solutions similaires à celles proposées par l'Alliance canadienne. Nous avons observé que la PC/RD accueille favorablement les mesures les plus controversées comme l'arrestation préventive et l'audience d'enquête. Elle souligne également qu'il est justifié de donner des pouvoirs accrus aux organes de surveillance, de faciliter l'obtention des mandats et autorisations d'interception et d'octroyer des pouvoirs supplémentaires au Centre de la sécurité des télécommunications Canada (CSTC). Il faut dire que, tout comme l'Alliance canadienne et les autres partis de l'opposition, ce parti insiste lui aussi sur l'ajout de protections afin de limiter les abus du pouvoir exécutif lors de l'éventuelle application de la loi. L'ajout de dispositions de temporisations, qui obligerait le Parlement à se reprononcer sur la légitimité de ces pouvoirs après un délai d'expiration prévu, a été débattu comme l'instauration d'une structure de surveillance continue. Malgré les protections suggérées, on voit tout de même comment ces deux partis conservateurs se représentent le 
terrorisme comme un problème avant tout individuel. On voit comment cette perception du problème les amène à soutenir C-36, ses mesures les plus controversées et même à proposer d'autres avenues sécuritaires qui, si non adoptées dans l'immédiat, devraient, selon eux, compléter ultérieurement la stratégie antiterroriste canadienne. Bref, les restrictions des droits et des libertés et l'orientation sécuritaire donnée à la lutte antiterroriste sont rendues acceptables et possibles en vertu d'une conception très individualisante de la menace terroriste.

Quant au gouvernement libéral, il insiste aussi sur le fait que ce projet de loi vise les gens qui commettent des activités terroristes, les gens qui les facilitent et qui les financent, prétendant ainsi agir à la source du problème. Toutefois, comme le suggère un membre du Parti libéral « [1]e projet de loi s'attaque directement aux causes profondes de la haine et de son expression [...] [en permettant aussi] aux tribunaux d'ordonner la suppression de la propagande haineuse diffusée publiquement par des moyens informatiques [...] » (Gurbax Malhi, Libéral, Ibid.: 6240). De plus, il crée une nouvelle infraction de méfait pour des actes commis contre un lieu de culte et motivés par la haine ou par des préjugés. Ainsi, selon la position libérale ici défendue par Gurbax Malhi, le « [...] projet de loi fait tout ce qu'il faut pour arrêter les terroristes et faire cesser les actes terroristes, pour combattre la haine qui engendre le terrorisme, et pour protéger nos valeurs et notre style de vie »(Gurbax Malhi, Libéral, Idem). Notre analyse révèle toutefois que tous les membres du caucus libéral ne se sont pas rangés derrière cette position. Certains ont tenté de faire valoir des pistes de solutions qui, selon eux, devraient être débattues et ne devraient pas être écartées. Si ces dernières n'ont pas été intégrées au projet de loi porté par le gouvernement, ils évoquent une définition de la problématique terroriste différente et proposent une réponse tout aussi différente que celles analysées jusque-là. Parmi les aspects évoqués figurent les conditions socio-économiques qui formeraient un terreau fertile du terrorisme:

Est-il trop tôt pour discuter de l'histoire des relations internationales et de la politique étrangère dans un contexte plus large? Est-il trop tôt pour établir un dialogue sur les responsabilités de chacun d'entre nous en tant que citoyens du monde? Pour vaincre le terrorisme, nous devons discuter des causes profondes des activités terroristes et nous y attaquer. Il est question des iniquités qui sont la source de mécontentement. Nous devons reconnaître les disparités politiques et économiques qui entraînent un vide socio-culturel et la famine dans certaines régions du monde (Stan Keyes, Libéral, Ibid.: 6214).

Andrew Telegdi, du Parti libéral, poursuit en ce sens :

L'éradication du terrorisme sera une tâche longue et pluridimensionnelle, et c'est pourquoi nous devons nous efforcer d'éliminer les conditions propices à l'émergence du terrorisme. [...] Il faut aussi réduire les différences de richesse entre les habitants de l'Ouest et ceux du reste du village planétaire. Notre sécurité et notre bien-être ne dépendant pas que de la création et du maintien de conditions politiques et sociales, d'institutions qui peuvent se maintenir et jouissent de la confiance de ceux qu'elles doivent servir. L'Ouest doit aussi consentir des ressources accrues pour le développement humain dans les sociétés troublées, si nous voulons combattre efficacement le terrorisme (Andrew Telegdi, Libéral, Ibid: 6230).

Toutefois, malgré une tentative de définir le problème du terrorisme et ses causes profondes en termes de conditions sociales, "d'iniquités à la source de mécontentements », de " différences de richesse entre les citoyens du monde », etc., c'est un problème et des solutions définies comme individualisées qui dominent de manière générale les discours des parlementaires libéraux, alliancistes et conservateursprogressistes. Leurs solutions, plutôt que de travailler à réduire le mécontentement, d'agir sur les conditions sociales créatrices de misère et d'injustices ou d'encourager le développement humain des sociétés troublées, répondent de manière individualisante à un problème défini comme individuel. Cette construction du risque les mène à privilégier notamment des pouvoirs accrus aux forces de l'ordre et aux 
ministères, un accès restreint à la libération conditionnelle, des peines plus sévères et consécutives, la criminalisation de comportements supplémentaires (comme l'activité terroriste et la facilitation d'une telle activité, par exemple) comme moyens efficaces de combattre le terrorisme, solutions d'autant plus envisagées lorsqu'il s'agit, dans leurs discours, de protéger les Canadiens « innocents » contre ces « autres ».

Les députés du BQ et du NPD proposent, quant à eux, une définition élargie du problème. Bien évidemment, ils font peser sur les individus, les « terroristes responsables » une grande partie de la responsabilité et du problème terroriste. Par contre, nous remarquons une tendance accrue, au fur et à mesure de l'avancement des débats, à questionner la nécessité de certains articles prévus dans le projet de loi C-36 et à remettre en cause leur efficacité à toucher aux causes profondes du terrorisme.

Puisqu'il est possible d'améliorer la sécurité des Québécois et des Canadiens en appliquant d'une manière rigoureuse les mesures de sécurité existantes, est-il nécessaire d'aller aussi loin que de modifier le Code criminel pour donner plus de pouvoir à la police dans le domaine de la détention préventive, de la surveillance électronique ou de la lecture du courrier postal ou électronique? [...] Enfin, il faut s'interroger aussi sur l'efficacité à long terme de la stratégie globale proposée. Croit-on vraiment qu'avec les frappes aériennes américaines, on pourrait, avec une loi antiterroriste, empêcher de tels actes soient posés dans l'avenir? Il faut aller au-delà des solutions qui sont uniquement militaires et policières. Il faut apporter une solution politique au problème (Christiane Gagnon, BQ, Ibid.: 6241).

Des solutions politiques sont d'ailleurs proposées par un membre du Bloc québécois :

Je peux comprendre des pays comme l'Afghanistan, qui vivent dans un état de pauvreté épouvantable. La racine de l'intégrisme est sûrement alimentée par des problèmes sociaux. Quand on voit que là-bas, des petites filles n'ont pas accès à l'éducation, des garçons non plus, que des gens n'ont pas accès à une vie épanouie, qu'ils n'ont pas accès à un degré légitime de richesse, qu'ils n'ont pas accès à des biens de base, il faut s'attendre à ce que, à un moment donné, cela saute. Cela a toujours été ma thèse, que j'ai probablement tirée de gens comme Nelson Mandela qui disait : «La sécurité pour quelques-uns, c'est l'insécurité pour tous. » [...] Au sujet de l'accès à l'éducation, je suis un de ceux qui croient qu'un des antidotes aux problèmes de ce monde, à la pauvreté et au terrorisme, c'est l'éducation (Stéphan Tremblay, BQ, Hansard, 18-10-2001: 6298).

Bien évidemment ces composantes définitionnelles du terrorisme se heurtent à celles d'autres partis comme l'Alliance canadienne qui a ouvertement remis en question l'idée que la pauvreté puisse être l'une des causes du terrorisme : "Il n'était pas question de pauvreté. Oussama ben Laden possède des millions de dollars. Les talibans sont un groupe corrompu qui s'est enrichi grâce aux profits de la vente d'héroïne, accumulés depuis des années pour financer ses efforts de guerre et sa guérilla » (Keith Martin, AC, Ibid. : 6307). Malgré les désaccords, l'analyse montre que le BQ, le NPD et, à quelques reprises les Libéraux, ont tenté d'élargir la définition du risque au-delà des individus « terroristes responsables ». En effet, les positions défendues par ces trois partis politiques tiennent compte de nombreux facteurs qui alimenteraient potentiellement le terrorisme, comme la pauvreté, les lacunes en éducation et les inégalités économiques entre les pays. Toutefois, cet effort de ne pas restreindre la définition du problème qu'à de simples individus, plus ou moins organisés et financés, n'a pas été intégré au projet de loi C-36, outre les dispositions abordées précédemment, qui visent la criminalisation de gestes haineux et le pouvoir de retirer des propos haineux d'Internet.

Ainsi, la sélection d'une solution au problème du terrorisme, liée à sa manière particulière de le définir, émerge au cours des débats. Le projet de loi antiterroriste en arrive à privilégier et à encastrer une stratégie sécuritaire générale que l'on peut qualifier d'individualisante, car elle vise principalement des individus identifiables qui facilitent, financent, complotent, planifient ou commettent des activités dites terroristes. Il s'agit d'une solution qui prône notamment la criminalisation et qui privilégie différents 
pouvoirs de recherche, d'enquête et de prévention, tels que nous avons vu plus tôt. Ces solutions conduisent à une individualisation du problème. L'institutionnalisation de cette solution reflète une perspective avant tout libérale-conservatrice et rejette une conception plus large du projet, soutenue par le BQ, le NPD et quelques rares membres du Parti libéral. Les contre-discours du BQ et NPD définissent l'acte terroriste comme le symptôme d'iniquités socio-économiques plus profondes qui, selon eux, demandent à être traitées. Le fait que le projet de loi C-36 ne prenne pas en considération les conditions qui favoriseraient le terrorisme et mette de côté la conception que s'en font le BQ et le NPD, explique pourquoi le NPD l'a rejeté en deuxième lecture et le $\mathrm{BQ}$ en troisième lecture.

En refusant de considérer la source des problèmes ailleurs que dans des individus « terroristes », il a été plus difficile pour ces partis de percevoir comme légitimes l'octroi de nouveaux pouvoirs policiers et ministériels intrusifs et les restrictions de droits et libertés qui pourraient s'en suivre. De plus, tant le BQ que le NPD considéraient C-36 davantage comme un projet de loi qui avait comme objectif de rassurer les Canadiens, nos alliés politiques, économiques et la communauté internationale. Il s'agissait ainsi, selon eux, d'un moyen pour le gouvernement canadien de montrer qu'il était proactif, plutôt que de constituer des mesures adéquates au regard des sources du problème. Enfin, les membres des Caucus bloquistes et du NPD ont estimé que la nécessité de restreindre les droits et les libertés n'a pas été démontrée par les Libéraux. Comme le remarque Svend Robinson,

Avant d'invoquer ces nouveaux et vastes pouvoirs que prévoit le projet de loi C-36, il est extrêmement important que le gouvernement apporte aux Canadiens la preuve que les pouvoirs déjà accordés à la Gendarmerie royale du Canada, au SCRS, au Centre de la sécurité des télécommunications et aux Forces armées du Canada ne suffisent pas face à la menace terroriste (Svend Robinson, NPD, Hansard, 16-10-2001: 6247).

Pour le BQ comme pour le NPD, l'augmentation de la sécurité ne se résume pas à l'adoption d'une panoplie de mesures visant à cibler des individus considérés comme dangereux, mais repose également sur une amélioration des conditions socio-économiques qui, selon leurs dires, pousseraient des individus à devenir des terroristes.

Mais pourquoi la vision conservatrice-libérale du problème a-t-elle été priorisée dans C-36 à l'encontre de ces contre-discours? Il faut comprendre que l'environnement politique dans lequel se sont joués ces luttes pour la « sécurisation antiterroriste » est caractérisé par des rapports de force propres à un gouvernement majoritaire libéral qui influent sur la capacité même d'imposer un autre point de vue. En effet, les règlements de l'institution parlementaire canadienne octroient aux partis du temps de parole et un nombre de votes proportionnels au nombre de sièges remportés lors de l'élection. Fort de sa position majoritaire, le Parti libéral pouvait faire adopter son projet de loi sans négocier avec l'opposition. Notre examen des amendements apportés au projet de loi antiterroriste confirme que les Libéraux ont utilisé leur majorité pour décider de l'adoption de C-36, de sa teneur et donc de la stratégie sécuritaire générale qu'elle contient. Lors de l'étude article par article du projet de loi devant le Comité de la justice et des droits de la personne (JUST), un amendement au projet de loi a été concédé au BQ, un à l'Alliance canadienne, dix à la PC/RD (qui étaient majoritairement des concordances entre la version française et anglaise) et aucun au NPD. Pendant cette même réunion, le gouvernement Libéral a adopté, à lui seul, 122 amendements desquels au moins 28 ont fait l'objet d'une dissidence officielle. Le déséquilibre perçu entre les voix discordantes et le point de vue libéral a provoqué l'ire des députés du BQ, qui ont relevé un "manque de sérieux » de l'étude (Michel Bellehumeur, BQ, Hansard, 26-11-2001 : 7476). Lors de l'étude du rapport à la Chambre des communes, neuf amendements ont été rejetés, un seul a été accepté et, en troisième lecture, les trois amendements proposés seront écartés du revers de la main. Au Sénat, les sept amendements conservateurs proposés en comité seront rejetés, tout comme les cinq autres qui seront proposés en troisième lecture.

On peut donc constater que la sélection de la stratégie sécuritaire présente dans C-36 s'est faite au profit de la perspective individualisante défendue par le Parti libéral qui a trouvé écho dans les rangs des partis conservateurs. Il est donc pertinent de se demander quelles sont les conséquences d'une telle sélection sécuritaire individualisante? Ici, nous quittons le registre du contenu des discours parlementaires pour poser 
la question des effets de pouvoir potentiels de ces discours présentant cette stratégie sécuritaire individualisante. Pour ce faire, nous revenons au champ des «études critiques de la sécurité » pour proposer une série d'hypothèses sur les effets de pouvoir que peuvent potentiellement occasionner ces discours sécuritaires individualisants institués par C-36.

\section{Réflexions sur les effets de pouvoir de cette sélection sécuritaire}

D'abord, il saute aux yeux que le discours de la sélection sécuritaire prévue dans C-36 a un effet majeur, soit celui de limiter le regard des parlementaires, de l'État et des Canadiens à l'individu terroriste et à son réseau, empêchant ainsi un regard analytique qui voudrait poser comme problématiques les conditions sociales de pauvreté, d'exclusion et d'inégalités mondiales ou qui chercherait à solutionner ces dernières dans un effort antiterroriste. Notre analyse ne vise pas à confirmer ou à infirmer qu'il existe objectivement un lien causal notamment entre les inégalités sociales et le terrorisme, ni à dire que telle ou telle manière de définir le terrorisme présentée dans les discours par un parti ou un autre est vraie ou fausse, fondée scientifiquement ou non, voire même qu'on devrait privilégier une définition au détriment d'une autre. Plutôt, à l'instar du théorème de Thomas, qui soutient que « (q)uand les hommes considèrent certaines situations comme réelles, elles sont réelles dans leurs conséquences » (Thomas et Thomas, 1932 cité dans Delas et Milly, 2010: 432), nous soutenons que, dès que certains parlementaires définissent des éléments à caractère social comme réellement problématiques pour eux, cette construction sociale du problème devient réelle dans ses conséquences. Nous avons déjà vu comment ces perceptions du problème du terrorisme ont favorisé soit un vote parlementaire contre le projet de loi ou contre certaines des mesures sécuritaires proposées en son sein. À l'inverse, les discours individualisants sur le terrorisme sont tout aussi réels dans leurs conséquences pour d'autres parlementaires qui appuient le projet de loi et plusieurs de ses mesures. Une fois institués par C-36, nous postulons que ces discours individualisants peuvent potentiellement avoir des effets de pouvoirs sur l'État et ses appareils, qui doivent prendre cette loi comme une directive et doivent structurer leurs propres discours et pratiques en conséquence. Mais nous croyons également à la possibilité d'effets de pouvoir de ces discours individualisants institués sur la manière dont les Canadiens perçoivent le problème du terrorisme et sa solution. En effet, étant une technologie par laquelle on tente de démontrer la certitude, l'autorité et la légitimité (Ericson, 2007 : 18), la loi impose une vision du problème particulière et encourage l'État, ses appareils et les Canadiens à se rallier à ces définitions et solutions. C'est en ce sens que nous pouvons affirmer que la sélection sécuritaire opérée par et dans C-36 a pour effet de limiter le regard des parlementaires, de l'État, mais aussi des Canadiens à l'individu terroriste, son réseau et d'exclure des éléments à caractère socio-économique de la réflexion. En effet, la loi parle avec autorité et tranche. Ce faisant, elle participe activement à la structuration de la manière dont les Canadiens pensent le problème du terrorisme. Une analyse complète de la réception et des réactions des différents acteurs à cette stratégie sécuritaire reste à faire, car nous ne postulons en aucun cas que tous les Canadiens acceptent de facto cette tentative de structuration. Nous voyons ici, dans cette stratégie sécuritaire, une tentative particulière d'instituer un regard sur le terrorisme et une solution au niveau de différents acteurs.

De plus, alors que C-36 propose surtout de criminaliser des individus « terroristes », il exclut une réflexion sur les mécanismes qui pourraient rassurer la collectivité sans un recours systématique aux institutions coercitives (comme le droit, la police ou les services de renseignement). Par exemple, qu'en est-il du recours aux discours rassurants, aux pratiques qui visent le développement d'un sentiment de courage envers le risque ou aux pratiques qui cherchent à corriger des facteurs qui alimenteraient les problèmes socio-économiques? Cet effet d'exclusion de la stratégie sécuritaire prévue par C-36 nous pousse à nous interroger sur l'état de la situation canadienne actuelle. Est-ce que la stratégie sécuritaire antiterroriste canadienne se limite à une telle stratégie individualisante? $\mathrm{Ou}$, au contraire, le Canada a-t-il mobilisé, avant ou après les attentats du 11 septembre 2001, des éléments d'une pratique qui définissent comme problématiques les inégalités socio-économiques ou certaines sources de mécontentement qui alimenteraient, aux dires de certains, le terrorisme? Notre objectif n'est pas de faire ici une analyse et un inventaire complet de ces initiatives, mais nos résultats de recherche nous invitent à poser cette question du potentiel déploiement de stratégies qui permettraient de rassurer la collectivité autrement que par le droit et 
les institutions coercitives. Notre objectif n'a pas été non plus de faire une analyse exhaustive de la nature du lien existant entre les conditions socio-économiques et le terrorisme. Plutôt, nos résultats démontrent, par une analyse de discours, le déploiement d'une stratégie sécuritaire et d'un discours essentiellement individualisants en C-36 qui sont confrontés à un contre-discours qui problématise différemment le terrorisme. Nous avançons que ces discours sécurisants, peu importe leur fondement (criminologique, psychologique, sociologique, politique, historique, etc), participent différemment à la construction de la problématique et des solutions au terrorisme. Ainsi, ceux-ci auraient des effets de pouvoir différents. L'un de ces discours étant insitutionnalisé, il reçoit un sceau de légitimité supplémentaire, ce dont le contrediscours ne bénéficie pas.

Outre le fait que le discours institué par cette stratégie sécuritaire a pour effet de détourner le regard de la problématisation des conditions sociales, économiques et politiques, avec ou sans raison, on constate qu'il a également pour effet d'empêcher une réflexion approfondie sur la construction socio-politique de ce qu'est le terrorisme. Qui définit le comportement de qui comme étant terroriste? En utilisant des notions de base en sociologie de la déviance et la théorie de l'étiquetage, on comprend que l'État appose une étiquette négative de « terroriste » pour condamner certains gestes violents qu'il n'approuve pas. Sans entrer dans le détail de ce qui constitue une " activité terroriste » selon le projet de loi, l'État aurait théoriquement la légitimité d'étiqueter certaines formes de violence de «terrorisme », car il détiendrait ce que Max Weber a nommé le «monopole légitime de la violence ». L'État déciderait donc de la violence acceptable, des acteurs qui peuvent l'utiliser (la police, l'armée, sécurité privée, etc.) et à quelles conditions ils peuvent l'utiliser. Cette question du monopole légitime de la violence de l'État doit être interrogée en tant qu'elle ne problématise pas les situations où un gouvernement utilise lui-même certaines formes de violences contre sa propre population ou celle d'un autre gouvernement. Il faut dire que ce sont les États eux-mêmes qui fixent les formes légitimes de la violence; dans cette perspective, peu s'autoproclament « terroristes ». Ce monopole de la violence ou, du moins, la nature construite du terrorisme ne peut pas être plus visible que lorsque C-36 donne le pouvoir, au gouverneur en conseil, de dresser une liste d'entités terroristes et définit ce qu'est une activité terroriste pour la première fois au Canada. En fait, une large proportion des débats sur C-36 s'est déroulée autour de la définition d'activité terroriste proposée sur laquelle se fondait la majorité des pouvoirs supplémentaires accordés par la loi. Tous les partis ont émis des préoccupations face à une définition qu'ils jugent trop large et trop floue. Certains parlementaires ont également estimé qu'elle risquait d'inclure des activités de revendication légitimes, ainsi que des usages de la violence dans le cas de luttes armées contre des régimes tyranniques. En effet, lors des débats, plusieurs parlementaires ont fait référence à Nelson Mandela et au Congrès national africain (ANC) qui ont utilisé la lutte armée contre le régime raciste et discriminatoire que constituait l'Apartheid. Ces interventions avaient pour objectif d'ébranler la confiance en l'objectivité perçue du libellé « activité terroriste » et de souligner la construction politique d'une telle catégorie. À l'époque, Mandela a été défini et étiqueté par certains États comme un «terroriste », mais ces parlementaires rappellent qu'il a été, par la suite, élu démocratiquement Président de l'Afrique du Sud. Ainsi, les discours qui individualisent le problème du terrorisme, institués dans C-36, ont pour effet de détourner l'attention de la qualité construite du terrorisme, plutôt que de présenter la complexité des processus par lesquels ces «terroristes »sont désignés comme tels. Bigo nuancerait certes le concept de Weber en disant que les politiciens ne sont plus les seuls à discourir sur la question des insécurités et donc à participer à la construction du terrorisme (2005). En effet, les experts et les professionnels du champ de la sécurité compétitionnent eux aussi dans des luttes discursives pour « le monopole du savoir légitime » sur l'insécurité et sur la sécurité. Bien que notre étude se concentre sur les parlementaires, Bigo nous rappelle l'importance d'analyser l'ensemble des luttes discursives entre acteurs tout comme la multiplicité des pratiques en situations d'insécurité (2005). En d'autres termes, le discours de la stratégie sécuritaire individualisante institué par C-36 aurait potentiellement pour effet de détourner le regard de l'État et de ses appareils, ainsi que celui des Canadiens de la qualité construite du terrorisme. Encore une fois, il reste à voir comment ont été reçues, in situ, ces tentatives d'institution d'un regard sécuritaire particulier par les différents acteurs.

Ainsi, on voit bien comment, d'une part, la sélection sécuritaire individualisante présente dans C36 semble avoir pour effet d'exclure une réflexion plus large sur les inégalités sociales dans le « village 
planétaire » qui peuvent potentiellement alimenter le terrorisme et, d'autre part, avoir pour effet d'exclure une réflexion sur la construction socio-politique de la catégorie «terroriste ». Un troisième effet du discours de la stratégie sécuritaire individualisante semble découler du deuxième. En occultant la construction sociopolitique de ce qu'est le terrorisme, la stratégie sécuritaire ne semble pas encourager une véritable réflexion sur la légitimité de la violence utilisée par les «terroristes » et les motifs qui le poussent à agir. S'est-on vraiment arrêté pour réfléchir aux attentats du 11 septembre 2001? Quels en étaient les motifs? Pourquoi les États-Unis ont-ils été ciblés ? Les États-Unis et le Canada avaient-ils un rôle à jouer ou une part de responsabilité quelconque? Si oui, lesquels? Par exemple, à l'instar de Baudrillard, on peut se demander si c'est « (...) cette superpuissance mondiale (...) qui, de par son insupportable puissance, a fomenté toute cette violence infuse de par le monde, et donc cette imagination terroriste (sans le savoir) qui nous habite tous » (2002: 10-11)? Baudrillard va même jusqu'à soutenir que :

Quand la situation est ainsi monopolisée par la puissance mondiale, quand on a affaire à cette formidable condensation de toutes les fonctions par la machinerie technocratique et la pensée unique, quelle autre voie y a-t-il qu'un transfert terroriste de situation? C'est le système luimême qui a créé les conditions objectives de cette rétorsion brutale. En ramassant pour lui toutes les cartes, il force l'Autre à changer les règles du jeu. Et les nouvelles règles sont féroces, parce que l'enjeu est féroce (Baudrillard, 2002: 15).

Cet auteur fait clairement un lien entre l'environnement international dans lequel nous évoluons et le terrorisme. Il estime ainsi que «(1)e terrorisme est immoral. L'événement du World Trade Center, ce défi symbolique est immoral, et il répond à une mondialisation qui est elle-même immorale » (Baudrillard, 2002: 20). Cependant, notre argument ici ne va pas aussi loin que celui de Baudrillard qui cherche à convaincre que l'islam serait devenu, ni plus ni moins, « le front mouvant de cristallisation de cet antagonisme » (2002: 23), qui serait en chacun de nous et qui prolongerait, à sa manière, la contestation communiste à l'hégémonie de la puissance libérale qui a triomphé mondialement suite à la chute du mur de Berlin. Notre argument affirme plutôt que C-36 et son discours semblent avoir pour effet de présenter à l'État, à ses appareils et aux Canadiens, des cibles floues en la personne d'individus terroristes et de leurs réseaux, alors que restent dans l'ombre la question de la légitimité de la violence et des motifs sous-jacents. L'introspection a eu lieu dans la mesure où les Libéraux se sont demandés ce que le Canada devait faire pour gérer le problème terroriste, plutôt que s'interroger sur les pratiques quotidiennes canadiennes et leurs impacts potentiels. Il faut dire que la rédaction du projet de loi de 175 pages en 34 jours et son adoption en 61 jours témoignent davantage d'importantes pressions environnantes et d'insécurités perçues que d'une véritable occasion d'introspection.

Alors, si le discours de la stratégie sécuritaire individualisante prévue dans le projet de loi antiterroriste canadien semble avoir des effets de pouvoir qui excluent des réflexions et détournent le regard, notamment des conditions socio-économiques qui entourent potentiellement les gestes de violence, de la construction politique de ce qu'est un terroriste et de la légitimité de la violence, à quoi cette stratégie sécuritaire individualisante est-elle utile? En effet, autant ces discours individualisants semblent présenter des effets « fantômes » qui laissent dans l'ombre plusieurs réflexions, autant ces discours semblent avoir des effets de pouvoir «producteurs ». Si nous avons déjà évoqué l'effet de produire et construire la problématique terroriste de manière particulièrement individualisante pour l'État, ses appareils et les Canadiens, on peut également penser que C-36, comme solution particulière, en vient à produire un certain sentiment de sécurité chez ceux qui adhèrent à ce discours individualisant. Sans prétendre faire le tour de la question et vérifier concrètement comment se matérialise ce sentiment de sécurité, nous posons l'hypothèse que ce sentiment de sécurité se concrétise différemment chez les gens qui voient les limites définitionnelles de ce discours individualisant. Le discours institué par C-36 constituerait ainsi autant un parapluie rassurant pour les alliés politiques et économiques du Canada que pour les Canadiens qui partagent cette définition individualisante du terrorisme.

Cependant, les discours institués dans C-36 semblent avoir pour effet de faire exister la problématique du terrorisme et de lui donner une crédibilité. Par sa forme légale, ce discours, qui est à la 
fois technologie, se réclame légitime. Ainsi, dans le même mouvement que le discours cherche à rassurer en proposant des solutions individualisantes, il fait exister objectivement la problématique nécessitant cette sécurisation. En ce sens, autant la solution repose sur un sentiment d'insécurité, autant elle reconfirme le problème défini initialement. En participant à sa définition, en la diffusant, en la présentant, en mobilisant l'agenda législatif et en bloquant celui-ci pendant un temps sur cette problématique, C-36 et ses discours seraient autant d'instances de création et de reproduction du problème terroriste. Un des effets de pouvoir de ces discours ne serait donc pas tant de protéger la population, ni de la rassurer, mais de produire une certaine forme de sentiment d'insécurité, condition sine qua non de la sécurisation et de sa légitimation. Ainsi, de la même manière que la prison crée, selon Foucault (1975/1998), la criminalité à laquelle elle dit vouloir mettre un terme, C-36 produirait des individus différents, terroristes, mauvais, ennemis de la nation, qui sont non confinés par des frontières géographiques et contre qui nous devons nous rallier, nous protéger. Ainsi, nous pouvons nous demander à quel prix C-36 et ses discours insécurisent-t-ils pour sécuriser?

Si la section précédente offre un début de réflexion sur quelques effets de pouvoir des discours individualisants institués par C-36, nous comprenons que cette réflexion ne dit rien sur la négociation in situ de ces exercices de pouvoir par les différents acteurs comme l'État, ses appareils et les Canadiens, par exemple. Il s'agit d'une série d'hypothèses provisoires, inspirées par le courant des « études critiques de la sécurité ", qui méritent d'être complétées, spécifiées et situées dans des travaux ultérieurs, mais qui permettent, d'ici là, d'entamer une réflexion provisoire sur les effets de pouvoir des discours de la sélection sécuritaire privilégiée dans C-36.

\section{Conclusion}

L'objectif de notre recherche était d'abord d'identifier comment les parlementaires canadiens ont défini la problématique terroriste dans le cadre des débats sur le projet de loi antiterroriste canadien et comment ceci cadrait leur réponse à cette problématique. Nous avons constaté que l'Alliance canadienne et la PC/RD se sont limités à une définition du problème individualisante, ce qui a contraint leur réponse individualisante, autorisant l'octroi de pouvoirs plus importants aux forces de l'ordre et aux ministères pour cibler les individus malfaiteurs, malgré les restrictions plus importantes des droits et libertés que cela implique. Ils ont cherché à promouvoir cette définition pour que soient adoptées des mesures sécuritaires plus importantes que prévues dans C-36. Les Libéraux, quoiqu'ils cherchent à adopter des mesures contre la haine et la discrimination et qu'ils problématisent discursivement les conditions sociales qui participent au problème, n'intègrent pas cette conception sociale à C-36. C'est en définissant le problème de manière plus large que le BQ et le NPD en arrivent à voter contre la solution législative qui ne répond pas, quant à eux, aux causes profondes du problème. Nous avions également comme objectif d'entamer une réflexion sur les effets de pouvoir des discours institués par C-36. Pour ce faire, nous avons démontré que la stratégie sécuritaire individualisante sélectionnée détournait la réflexion (1) des tensions socio-économiques qui peuvent potentiellement alimenter le problème; (2) de la construction socio-politique de ce qu'est le terrorisme; et (3) de la légitimité éventuelle des motifs derrière le terrorisme. Nous avons proposé que C36 et ses discours, bien qu'ils constituent un parapluie protecteur et rassurant pour les Canadiens, leurs alliés politiques et économiques sur la scène internationale, participent en même temps à la mise en forme individualisante du problème terroriste, à une certaine forme d'insécurisation, et donc à la construction de l'averse terroriste même que ces discours institués prétendent prévenir.

À propos de l'auteur : Pascal Dominique-Legault est un étudiant au doctorat en sociologie à l'Université Laval. Il peut être contacté au pascal.dominique-legault.1@ulaval.ca . L'auteur aimerait remercier le soutien de Line Beauchesne de l'Université d'Ottawa et celui du Conseil de recherches en sciences humaines (CRSH). 


\section{Références}

Baud, J. (2000). Défis du renseignement contemporain. Éthique publique, 2 (1), 151-157.

Baudrillard, J. (2002). L'esprit du terrorisme. Paris : Galilée.

Beauchamp, A. (1996). Gérer le risque, vaincre la peur. Montréal : Bellarmin.

Bigo, D. (2005). La mondialisation de l'(in)sécurité? Cultures \& Conflits, 58, Suspicion et exception, 53101.

Brodeur, J.-P. (2003). Bleu et gris: L'alliance méfiante. In J.P. Brodeur (Ed.), Les visages de la police (pp. 255-279). Montréal : Presses de l'Université de Montréal.

Canada (2001) Projet de loi C-36 : Loi modifiant le Code criminel, la Loi sur les secrets officiels, la Loi sur la preuve au Canada, la Loi sur le recyclage des produits de la criminalité et d'autres lois, et édictant des mesures à l'égard de l'enregistrement des organismes de bienfaisance, en vue de combattre le terrorisme, Ottawa, Parlement du Canada, $37^{\text {ième }}$ législature, $1^{\text {èr }}$ session, sanctionné.

Canada, Parlement, Chambre des communes. Débats de Débats de la Chambre des Communes, compte rendu officiel (hansard), Ottawa, $37^{\text {ième }}$ législature, $1^{\text {ère }}$ session, vol 137 (095), 16 octobre 2001, $6250 \mathrm{p}$.

Canada, Parlement, Chambre des communes. Débats de Débats de la Chambre des Communes, compte rendu officiel (hansard), Ottawa, $37^{\text {ième }}$ législature, $1^{\text {ère }}$ session, vol 137 (096), 17 octobre 2001, $6291 \mathrm{p}$.

Canada, Parlement, Chambre des communes. Débats de Débats de la Chambre des Communes, compte rendu officiel (hansard), Ottawa, $37^{\text {ième }}$ législature, $1^{\text {ère }}$ session, vol 137 (097), 18 octobre 2001, $6357 \mathrm{p}$.

Canada, Parlement, Chambre des communes. Débats de Débats de la Chambre des Communes, compte rendu officiel (hansard), Ottawa, $37^{\text {ième }}$ législature, $1^{\text {ère }}$ session, vol 137 (118), 26 novembre 2001, $7529 \mathrm{p}$.

Delas, J. et B. Milly (2010). Histoire des pensées sociologiques. $3^{\mathrm{e}}$ édition, Paris : Armand Colin.

Delumeau, J. (1978). La peur en Occident (XIVe-XVIIIe siècles). Paris : Fayard, Pluriel.

Douglas, M. et A. Wildavsky (1983). Risk and Culture: An Essay on the Selection of Technical and Environmental Dangers. Berkeley: University of California Press.

Ericson, R.V. (2007). Crime in an Insecure World. Cambridge: Polity Press.

Foucault, M. (1975/1998). Surveiller et punir. La naissance de la prison. Paris : Gallimard.

Gross, O. (2001). Cutting Down Trees: Law-Making Under the Shadow of Great Calamities. In R.J.

Daniels, P. Macklem \& K. Roach (Eds.), The Security of Freedom-Essays on Canada's Anti-Terrorism Bill (pp. 39-61). Toronto: University of Toronto Press.

Haubrich, D. (2003). September 11, Anti-Terror Laws and Civil Liberties: Britain, France and Germany Compared. Government and Opposition, 38 (1), 3-28.

Huysmans, J. (2006). The Politics of Insecurity. Fear, Migration and Asylum in the EU. Londres: Routledge.

Kassimeris, G. (2007). Playing politics with terrorism: a user's guide. New York: Columbia University Press.

Landreville, P. (1986). Évolution théorique en criminologie: 1'histoire d'un cheminement. Criminologie, 19 (1), 11-31.

Macleod, A. (2004). Les études de sécurité : du constructivisme dominant au constructivisme critique. Cultures \& Conflits, 54, 1-24.

Martin, D., M. Fichelet et R. Fichelet (1977). Si la violence existe. Discours du violent. Déviance et Société, 1 (3), 291-308.

Mueller, J. (2006). Overblown: how politicians and the terrorism industry inflate national security threats and why we believe them. New York: Free Press.

Moffette, D. (2012). Études critiques de la sécurité : quelques contributions théoriques pour une anthropologie de la sécurité. Aspects sociologiques, 19 (1), 39-68. 
Vol. 2, Iss/Num. 2, Fall/Automne 2013

Thomas, W.I. et D.S. Thomas (1932). The Child in America: Behavior Problems and Programs. New York: Knopf.

Wark, W. (2004). Learning lessons (and how) in the war on terror. International Journal, 60 (1), 71-90.

Weinrib, L.E. (2001). Terrorism's Challenge to the Rule of Law. In R.J. Daniels, P. Macklem \& K. Roach (Eds.), The Security of Freedom-Essays on Canada's Anti-Terrorism Bill (pp. 93-108). Toronto: University of Toronto Press.

White House. Address to a Joint Session of Congress and the American People, Office of the Press Secretary, 20-09-2001. Consulté le 10-06-2013. URL: http://georgewbushwhitehouse.archives.gov/news/releases/2001/09/20010920-8.html 